\title{
Modeling and hybrid simulation of slow discharge process of adsorbed methane tanks
}

\author{
S.C. Hirata ${ }^{a}$, P. Couto ${ }^{\text {}}$, L.G. Lara $^{c}$, R.M. Cotta ${ }^{a}, *$ \\ ${ }^{a}$ Mechanical Eng. Dept., Universidade Federal do Rio de Janeiro, COPPE/UFRJ, Brazil \\ b Industrial Eng. Dept., Universidade Federal do Rio de Janeiro, POLI/UFRJ, Brazil \\ ${ }^{c}$ Petrobrás Petróleo Brasileiro S.A., IEABAST/EAB/ENPRO, Rio de Janeiro, Brazil
}

\section{A R T I C L E I N F O}

\section{Article history:}

Received 30 April 2008

Received in revised form 3 September 2008

Accepted 4 September 2008

Available online 15 October 2008

\section{Keywords:}

Methane

Natural gas

Adsorption

ANG

Hybrid methods

Integral transforms

\begin{abstract}
A B S T R A C T
The slow discharge process of a methane tank filled with porous carbonaceous adsorptive material is modelled and solved by the Integral Transform Method, yielding a hybrid numerical-analytical solution of the related energy equation. A transient one-dimensional nonlinear formulation is adopted, which includes the compressed and adsorbed gas thermal capacitances, the reservoir wall thermal capacitance effect and the gas compressibility influence. The overall mass balance is employed to determine the pressure field evolution, here assumed as spatially uniform. A thorough covalidation analysis is performed, with both numerical and experimental data available in the literature, and the relative importance of some terms in the energy equation formulation is inspected. Finally, different possibilities for the reduction of the adverse effect of the heat of adsorption on storage capacity are proposed and investigated.
\end{abstract}

(c) 2008 Elsevier Masson SAS. All rights reserved.

\section{Introduction}

Natural gas is becoming an economically attractive fuel for many industrial applications around the world. However, the use of natural gas is still limited since its storage and transportation are not convenient when compared to conventional liquid fuels. When natural gas is in the gas phase, transportation occurs in gas pipelines and storage is performed in high-pressure cylinders, as CNG (compressed natural gas) at pressure levels around 200 atmospheres. Although this technique provides storage capacity of 200 V/V (volume of stored natural gas, measured at Standard Pressure and Temperature conditions-STP, per unit storage volume), it requires reliable and safe cylinders as well as costly compressing equipment. If the natural gas is in the liquid phase as liquefied natural gas (LNG), volumes as large as $600 \mathrm{~V} / \mathrm{V}$ can be transported in ships and trucks. In this case, the gas must be cooled to a temperature as low as $-160^{\circ} \mathrm{C}$. This technique is highly energetically expensive as heat leaks during transportation and storage vaporizes the LNG. Also, to be reused, the gas must be vaporized in appropriate equipment.

Adsorbed natural gas (ANG) has recently emerged as an alternative to CNG and LNG, as observed by the growing number of scientific contributions in this direction [1-21]. In the case of natural gas, the gas molecules uptake process occurs at relatively low

\footnotetext{
* Corresponding author.

E-mail address: cotta@mecanica.coppe.ufrj.br (R.M. Cotta).
}

pressures, in the order of 35 to 50 atmospheres, which provides three main benefits: (1) compression costs reduction, (2) increased safety of the reservoir, and (3) design flexibility of the storage tanks. Despite of these benefits, the storage capacity of ANG reservoirs remains lower than LNG and CNG, being of the order of $90 \mathrm{~V} / \mathrm{V}$ [1] up to $164 \mathrm{~V} / \mathrm{V}$ [2]. The U.S. Department of Energy storage target for ANG vessels was set at $150 \mathrm{~V} / \mathrm{V}$ and it was later revised to $180 \mathrm{~V} / \mathrm{V}$.

According to Biloé et al. [3], the performance and viability of an ANG system depends closely on the micro-porous characteristics of the adsorbent as well as on the heat and mass transfer properties. The adsorption phenomenon that occurs during the charge of an ANG reservoir is an exothermic process. The temperature increase caused by the release of heat of adsorption, results in less stored methane capacity under dynamic conditions. During discharge of the ANG reservoir, the desorption process is endothermic which causes the adsorbent bed to cool down. As a result, some amount of gas is retained inside the reservoir at depletion pressure, reducing the delivered methane capacity. The thermal effects depend closely on the heat transfer properties of the adsorbent bed as well as on the external heat exchange at the vessel walls [4-6].

Although the literature presents suggestions to improve the storage capacity of ANG systems, the thermal control of an adsorptive reservoir was not yet fully explored. The addition of thermal energy to the adsorbent bed increases its temperature, enhancing the natural gas deliverance capacity during the discharge process. 
Based on a model previously established for the analysis of dynamic thermal processes in ANG tanks [5], a finite differences procedure was implemented for the two-dimensional analysis of the slow discharge process [18-20] and employed in the analysis of a heat pipe solution for its thermal control [21]. The present work will focus on the development of an error-controlled solution of the energy equation related to the slow discharge process of adsorbed methane in cylindrical tanks filled with porous adsorptive material. Pure methane is considered thus avoiding the consideration of additional effects related to higher hydrocarbons or impurities. The aim is to provide a benchmark solution for validation of such previous developments, while offering an alternative solution path for the inspection of the different modeling and formulations so far considered in the open literature, which are indeed not unanimous on which terms to account for. For this purpose, the Generalized Integral Transform Technique (GITT) [22-25] is employed in the hybrid numerical-analytical solution of the proposed one-dimensional transient formulation for the energy equation that governs the gas temperature inside the tank, including a fifth kind boundary condition that accounts for the wall thermal capacitance effect. The simplified one-dimensional model incorporates the major nonlinear heat and mass transfer effects present in the actual physical situation, while serving the purpose of offering a validation starting point for numerical solutions of more general multidimensional models. Besides, a number of situations dealing with cylinders of high aspect ratio, such as in applications that involve transportation of tanks beams $[5,18]$, the one-dimensional model already provides a fairly reliable representation of the involved phenomena. An implicit filtering procedure is devised to completely eliminate the transient operator from the boundary condition with thermal capacitance, relaxing the need for employing other more cumbersome convergence acceleration techniques. Also, in order to avoid a coupled coefficients matrix in the transient operator of the transformed energy equation, all the nonlinearities of the problem are moved into the general source term [26,27], which also includes the heat of adsorption and compressibility effects. The solution procedure was entirely developed in the Mathematica v. 5.2 system [28], including all of the symbolic computation steps required by the hybrid solution implemented. Numerical results are first critically covalidated with the previously developed two-dimensional finite differences solution $[18,20]$ and other numerical implementations available in the literature [5]. Our hybrid solution is also compared to the experimental data of [4]. Then, the relative importance of some terms in the energy equation formulation, as presented in different contributions in the literature, is critically inspected and the influence of a few effects that are often considered negligible is analysed. Finally, some solutions for the reduction of the adverse effect of the heat of adsorption on storage capacity are proposed.

\section{Problem formulation}

A nonlinear one-dimensional transient model for the discharge process of a cylindrical ANG vessel is presented, as represented in Fig. 1. A sufficiently long cylindrical vessel of external radius $R_{\text {ext }}$ and length $L$, with a wall thickness of $\delta_{w}$ is considered, neglecting axial temperature gradients for tanks with high aspect ratio. The gas flows outwards from the cylinder through a cylindrical opening at the origin of the system, at a constant mass flow rate. Heat transfer by convection $\left(h_{\infty}\right)$ between the external walls of the cylinder and the surroundings at $T_{\infty}$ is considered. The activated carbon bed is considered to be composed of activated carbon pellets with an effective porosity of $\varphi_{\text {eff. }}$. An axisymmetric temperature condition is considered at the centre of the cylinder.

The energy balance equation derived for the slow discharge process was obtained by writing the first law of thermodynam-
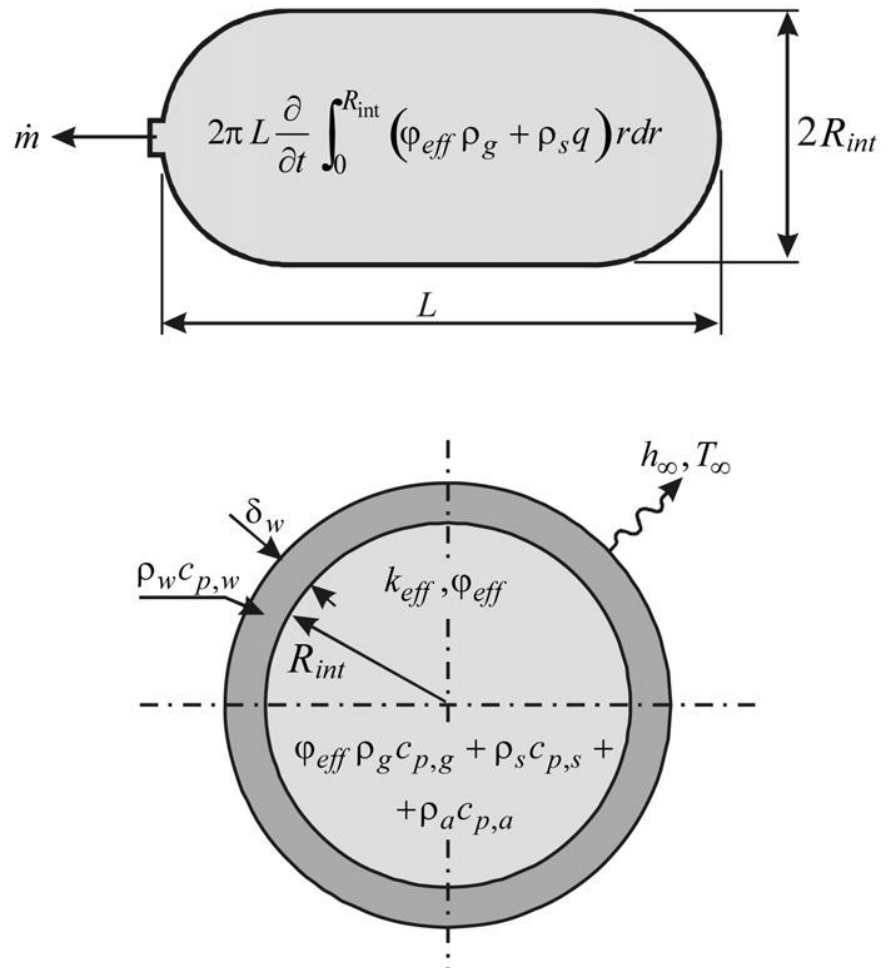

Fig. 1. Physical model for the mass and energy conservation equations.

ics for transient regime [18], accounting for the contribution of the solid phase (adsorbent), gas phase, and the adsorbed phase in the total energy stored in the reservoir. Also, the contribution of the energy transfer by heat diffusion in the solid phase is considered. The heat of adsorption is considered as a rate of internal heat generation. The assumptions made for the energy conservation equation derivation are: (1) instantaneous phase equilibrium between the adsorbed phase and the gas phase within the porous structure, (2) the local temperature of the adsorbent and free gas volume is the same due to a high intensity heat transfer between solid and gas phases. Also, (3) for the solid and adsorbed phases, the internal energy depends only on the local thermodynamic temperature and (4) the adsorbed phase is considered incompressible. As the discharge process is considered to be slow, (5) the pressure gradient inside the cylinder is negligible [5], i.e., the pressure is a function of time only. According to these assumptions, the energy equation is written as:

$$
\begin{aligned}
& \left(\varphi_{\mathrm{eff}} c_{p, g} \rho_{g}+\rho_{s} c_{p, s}+\rho_{s} c_{p, g} q\right) \frac{\partial T(r, t)}{\partial t} \\
& \quad=k_{\mathrm{eff}} \frac{1}{r} \frac{\partial}{\partial r}\left(r \frac{\partial T}{\partial r}\right)+\varphi_{\mathrm{eff}} \frac{d P(t)}{d t}-\rho_{s} \Delta H \frac{\partial q}{\partial t}
\end{aligned}
$$$$
\text { in } 0<r<R_{\text {int }}, t>0
$$

The left-hand side of Eq. (1) accounts for the energy stored in the control volume in the compressed gas within the pores of the porous structure, activated carbon bed and adsorbed phase, respectively. The first term in the right-hand side of Eq. (1) represents the heat diffusion in the activated carbon bed, while the last two terms represent the compressibility effect of the gas and the heat of adsorption. These two terms act as a heat source term in the energy equation. Initially, the system is considered to be at a homogeneous and uniform temperature and pressure, given by $T_{0}$ and $P_{0}$ respectively. The initial and boundary conditions of Eq. (1) are:

$T(r, 0)=T_{0}, \quad$ in $0<r<R_{\text {int }}$ 
$\frac{\partial T(0, t)}{\partial r}=0, \quad t>0$

$k_{\text {eff }} \frac{\partial T\left(R_{\text {int }}, t\right)}{\partial r}+\rho_{w} c_{p, w} \delta_{w} \frac{\partial T}{\partial t}=h_{\infty}\left(T_{\infty}-T\right)$

at $r=R_{\text {int }}, t>0$

Boundary condition (2c) accounts for the thermal capacity of the cylinder wall $\left(\rho_{w} c_{p, w}\right)$ and for the heat transfer by convection at the external wall of the cylinder. The heat transfer coefficient $h_{\infty}$ can be obtained from external convection correlations for horizontal/vertical cylinders available in the literature.

The mass conservation equation is given by:

$2 \pi L \frac{\partial}{\partial t} \int_{0}^{R_{\text {int }}}\left(\varphi_{\text {eff }} \rho_{g}+\rho_{s} q\right) r d r=\dot{m}$

The first term inside the integral represents the mass of compressed gas phase in the pores of the adsorbent bed, while the second term represents the mass of the adsorbed gas phase. Eq. (3) provides an expression for the total variation of the gas mass inside the reservoir as a function of time.

By using the ideal gas equation to correlate $P, T$ and $\rho_{g}$, Eq. (3) can be solved to determine the pressure history as a function of time $[P=f(t)]$ inside the cylinder for a given mass flow rate.

The amount of gas adsorbed by an adsorbent material when equilibrium is reached for a given temperature and pressure, is a function of the nature of the gas to be adsorbed and of the adsorbent material. For a given adsorbate-adsorbent system, the adsorption equilibrium relation is expressed by:

$q=f(P)_{T}$

where $q$ is the amount of gas adsorbed per unit mass of adsorbent (commonly in $\mathrm{kg}_{\text {gas }} / \mathrm{kg}_{\text {solid }}$ ). For the adsorption of natural gas, the gas is considered as uniform and homogeneous, with thermodynamic properties well defined by its temperature and pressure. Mota et al. [5] present the following experimentally obtained Langmuir equilibrium relation for the adsorption of methane in a G216 activated carbon bed:

$q=\left(q_{m} b P\right) /(1+b P)$

where $q_{m}=55920 T^{-2.3}$ and $b=1.0863 \times 10^{-7} \exp (806 / T)$.

\section{Solution methodology}

The Generalized Integral Transform Technique (GITT) is a hybrid numerical-analytical approach for nonlinear diffusion and convection-diffusion problems, which was originated from the classical integral transform method for linear transformable diffusion problems [23-25]. The basic concept is to propose eigenfunction expansions in all but one independent variable, which results in an analytical representation of the desired potential in all but this only one variable. Upon integral transformation of the original partial differential system, a coupled system of ordinary differential equations for the transformed potentials then results which is numerically solved to yield the expansion coefficients. The main features of this approach are the automatic error control procedure, the direct application to nonlinear formulations and irregular geometries, and the mild increase in computational cost for multidimensional applications. Its application to the present class of problems is particular motivating in light of the strong nonlinear nature of practically all of the terms and the relative importance of the source terms.

The first step in the application of the integral transform method in the present problem is to rewrite Eq. (1) so as to incorporate all of the nonlinear terms in the right-hand side of the energy equation, thus leaving the transient operator available for exact integral transformation, which avoids an implicit transformed system that would require matrix inversion for each step of the numerical integration of the transformed ODE system [26]. Since the adsorbed phase concentration, $q$, can be rewritten as a function of temperature and pressure, its derivative can be rewritten as:

$\frac{\partial q(T, P)}{\partial t}=\frac{\partial q(T, P)}{\partial T} \frac{\partial T(r, t)}{\partial t}+\frac{\partial q(T, P)}{\partial P} \frac{d P(t)}{d t}$

Then, Eq. (1) can be rewritten as:

$$
\begin{gathered}
\left(\varphi_{\mathrm{eff}} c_{p, g} \rho_{g}+\rho_{s} c_{p, s}+\rho_{s} c_{p, g} q+\rho_{s} \Delta H \frac{\partial q(T, P)}{\partial T}\right) \frac{\partial T(r, t)}{\partial t} \\
=k_{\mathrm{eff}} \frac{1}{r} \frac{\partial}{\partial r}\left(r \frac{\partial T}{\partial r}\right)+\left(\varphi_{\mathrm{eff}}-\rho_{s} \Delta H \frac{\partial q(T, P)}{\partial P}\right) \frac{d P(t)}{d t}
\end{gathered}
$$

in $0<r<R_{\text {int }}, t>0$

where the required derivatives of $q(T, P)$ are symbolically obtained from Eq. (5). After labeling the nonlinear term in the transient term as an effective thermal capacitance, $\rho c_{p, m}(T, P)$, the energy equation is given by:

$$
\begin{aligned}
\frac{\partial T(r, t)}{\partial t}= & {\left[k_{\text {eff }} \frac{1}{r} \frac{\partial}{\partial r}\left(r \frac{\partial T}{\partial r}\right)\right.} \\
& \left.+\left(\varphi_{\mathrm{eff}}-\rho_{s} \Delta H \frac{\partial q(T, P)}{\partial P}\right) \frac{d P(t)}{d t}\right] / \rho c_{p, m}(T, P)
\end{aligned}
$$

in $0<r<R_{\text {int }}, t>0$

The next step is the proposition of a filtering solution that explicitly accounts for the transient operator in the boundary condition (2c), that refers to the wall thermal capacitance effect. The temperature field is then written as:

$T(r, t)=T_{f}(r, t)+T^{*}(r, t)$

For the purpose of constructing a filter $T_{f}$, we consider a simple steady heat conduction formulation that obeys the original boundary conditions, in the form:

$$
\begin{aligned}
& \frac{\partial}{\partial r}\left(r \frac{\partial T_{f}}{\partial r}\right)=0, \quad \text { in } 0<r<R_{\text {int }} \\
& \frac{\partial T_{f}(0, t)}{\partial r}=0, \quad t>0 \\
& k_{\text {eff }} \frac{\partial T_{f}\left(R_{\text {int }}, t\right)}{\partial r}+\rho_{w} c_{p, w} \delta_{w} \frac{\partial T}{\partial t}=h_{\infty}\left(T_{\infty}-T_{f}\right) \\
& \text { at } r=R_{\text {int }}, t>0
\end{aligned}
$$

This implicit filter is readily obtained as:

$T_{f}(t)=T_{\infty}-\frac{\rho_{w} c_{p, w} \delta_{w}}{h_{\infty}} \frac{\partial T\left(R_{\text {int }}, t\right)}{\partial t}$

The proposed filter is $r$-independent and eliminates the transient operator in the boundary condition of the partial differential system, but it is not known a priori, since it depends on the unknown potential at the wall, $T\left(R_{\mathrm{int}}, t\right)$. Substitution of this filter into the original system, Eqs. (8), $(2 a-c)$, results in the filtered problem below:

$$
\begin{aligned}
\frac{\partial T^{*}(r, t)}{\partial t}= & \frac{1}{\rho c_{p, m}(T, P)}\left[k_{\mathrm{eff}} \frac{1}{r} \frac{\partial}{\partial r}\left(r \frac{\partial T^{*}}{\partial r}\right)\right. \\
& \left.+\left(\varphi_{\mathrm{eff}}-\rho_{s} \Delta H \frac{\partial q(T, P)}{\partial P}\right) \frac{d P(t)}{d t}\right]-\frac{d T_{f}(t)}{d t}
\end{aligned}
$$

in $0<r<R_{\text {int }}, t>0$

$T^{*}(r, 0)=T_{0}-T_{f}(0), \quad$ in $0<r<R_{\text {int }}$

$\frac{\partial T^{*}(0, t)}{\partial r}=0, \quad t>0$

$k_{\text {eff }} \frac{\partial T^{*}\left(R_{\text {int }}, t\right)}{\partial r}+h_{\infty} T^{*}\left(R_{\text {int }}, t\right)=0, \quad t>0$ 
Eq. (11) can be interpreted itself as the differential equation for $T_{f}(t)$ that couples with the above partial differential formulation, in the form:

$\frac{d T_{f}(t)}{d t}+\frac{\partial T^{*}\left(R_{\text {int }}, t\right)}{\partial t}=\frac{h_{\infty}}{\rho_{w} c_{p, w} \delta_{w}}\left(T_{\infty}-T_{f}(t)\right)$

with the initial condition

$$
\begin{aligned}
T_{f}(0)= & T_{\infty}-\frac{\rho_{w} c_{p, w} \delta_{w}}{h_{\infty}} \frac{1}{\rho c_{p, m}\left(T_{0}, P_{0}\right)}\left(\varphi_{\mathrm{eff}}-\rho_{s} \Delta H \frac{\partial q\left(T_{0}, P_{0}\right)}{\partial P}\right) \\
& \times \frac{d P(0)}{d t}
\end{aligned}
$$

The proposed eigenfunction expansion is based on the following eigenvalue problem:

$$
\begin{aligned}
& \frac{1}{r} \frac{\partial}{\partial r}\left(r \frac{\partial \psi_{i}(r)}{\partial r}\right)+\mu_{i}^{2} \psi_{i}(r)=0, \quad \text { in } 0<r<R_{\text {int }} \\
& \frac{\partial \psi_{i}(r)}{\partial r}=0, \quad r=0 \\
& k_{\text {eff }} \frac{\partial \psi_{i}\left(R_{\text {int }}\right)}{\partial r}+h_{\infty} \psi_{i}\left(R_{\text {int }}\right)=0
\end{aligned}
$$

where $\psi_{i}$ 's are the eigenfunctions, $\mu_{i}$ 's are the eigenvalues, and the normalization integral is written as:

$N_{i}=\int_{0}^{R_{\mathrm{int}}} r \psi_{i}^{2}(r) d r$

while the normalized eigenfunctions and eigenvalues are obtained from

$$
\begin{aligned}
& \tilde{\psi}_{i}(r)=\frac{\psi_{i}(r)}{\sqrt{N_{i}}}=\frac{J_{0}\left(\mu_{i} r\right)}{\sqrt{N_{i}}} \\
& -k_{\text {eff }} \mu J_{1}\left(\mu R_{\text {int }}\right)+h_{\infty} J_{0}\left(\mu R_{\text {int }}\right)=0
\end{aligned}
$$

The integral transform pair can then be defined as:

$$
\begin{aligned}
& \bar{T}_{i}(t)=\int_{0}^{R_{\text {int }}} r \tilde{\psi}_{i}(r) T^{*}(r, t) d r, \quad \text { transform } \\
& T^{*}(r, t)=\sum_{i=1}^{\infty} \tilde{\psi}_{i}(r) \bar{T}_{i}(t), \quad \text { inversion }
\end{aligned}
$$

The partial differential Eq. (12a) is now integral transformed, by operating on it with $\int_{0}^{R_{\text {int }}} r \tilde{\psi}_{i}(r) d r$, to yield:

$$
\begin{aligned}
& \frac{d \bar{T}_{i}(t)}{d t}=h_{i}(t)-f_{i} \frac{d T_{f}(t)}{d t}, \quad \text { for } i=1,2, \ldots, t>0 \\
& h_{i}(t)=\int_{0}^{R_{\text {int }}} r \tilde{\psi}_{i}(r) \frac{1}{\rho c_{p, m}(T, P)}\left[k_{\mathrm{eff}} \frac{1}{r} \frac{\partial}{\partial r}\left(r \frac{\partial T^{*}}{\partial r}\right)\right. \\
& \left.\quad+\left(\varphi_{\mathrm{eff}}-\rho_{s} \Delta H \frac{\partial q(T, P)}{\partial P}\right) \frac{d P(t)}{d t}\right] d r \\
& f_{i}=\int_{0}^{R_{\text {int }}} r \tilde{\psi}_{i}(r) d r \\
& \bar{T}_{i}(0)=\left(T_{0}-T_{f}(0)\right) f_{i}
\end{aligned}
$$

The coefficients vector $h_{i}(t)$ needs to be evaluated numerically due to the nonlinear nature of this term. Here, however, a semianalytical procedure was preferred [27], based on the exact integration of the periodic behavior of the eigenfunctions. For a given pressure evolution, system (16) is then joined with Eqs. (13) and

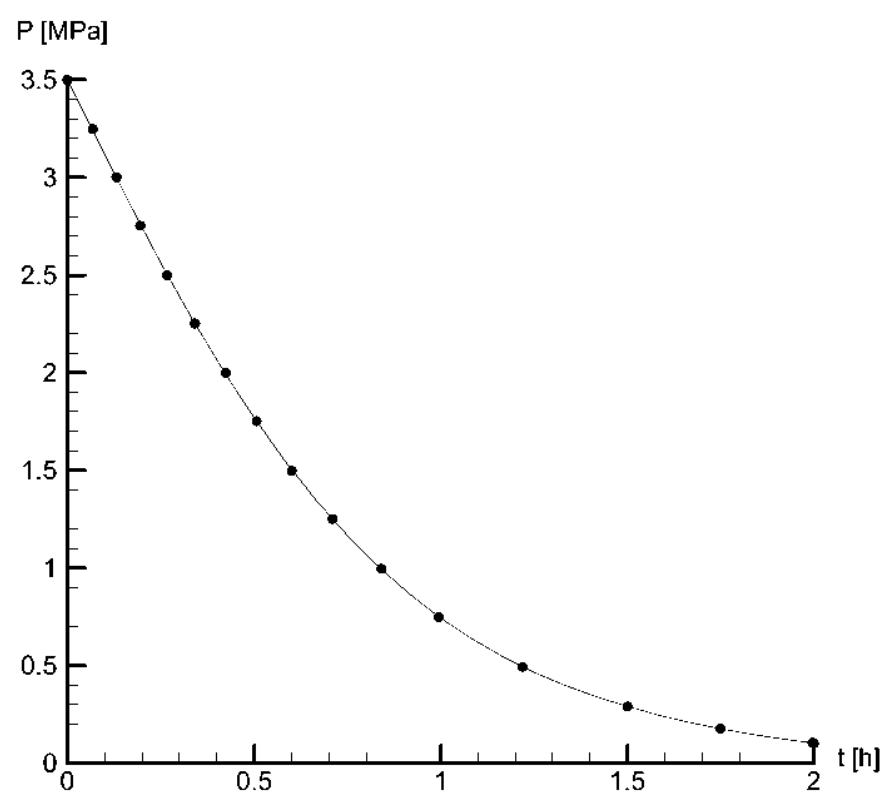

Fig. 2. Estimated discharge process pressure history for comparison with Mota et al. [5].

corresponding initial conditions, to form the transformed ODE system which is numerically integrated employing the NDSolve function of the Mathematica system [28]. The inversion formula (15b) followed by the filtering expression, Eq. (9), then recovers the desired temperature field in analytical form along the radial coordinate.

An iterative procedure is finally required for updating the pressure field evolution, $P(t)$. The continuity equation can be integrated by the same semi-analytical approach employed for the transformed system coefficients [27], yielding a differential equation for the pressure field in terms of the transformed potentials above described. The initial estimate for the pressure evolution is then provided by the isothermal discharge limiting solution.

\section{Results and discussion}

Before presenting and discussing some physical aspects of the problem, we first seek a verification of the constructed symbolic-numerical code and a demonstration of the performance of the proposed methodology. Mota et al. [5] presented a onedimensional transient theoretical analysis of the slow discharge of a 50 litres ANG cylinder, where emphasis was given to thermal and mass diffusion effects in the activated carbon bed. Natural gas was approximated as pure methane and modelled as ideal gas. Although Mota et al. [5] presented a complete energy equation, the pressure history was imposed, and not determined by the mass conservation equation. The imposed pressure history was expected to lead to a constant mass flow rate at the cylinder outlet, but this is not explicitly provided, which makes it more difficult to reproduce the results in their work. Nevertheless, due to the similarities between the two models, we have followed the work of Mota et al. [5] in this comparison effort. Therefore, for an estimated pressure history obtained from a sixth order polynomial fit, presented in Fig. 2, we have first compared the GITT solution with the numerical results of [5]. Fig. 3 shows various radial temperature profiles, for different times along the discharge process $(t=0.25,0.5,0.75,1.0,1.25,1.5,1.75,2.0$ hours). The arrow indicates increasing time steps and one may observe a quite reasonable agreement for shorter times and an increasing discrepancy for larger discharge times. 


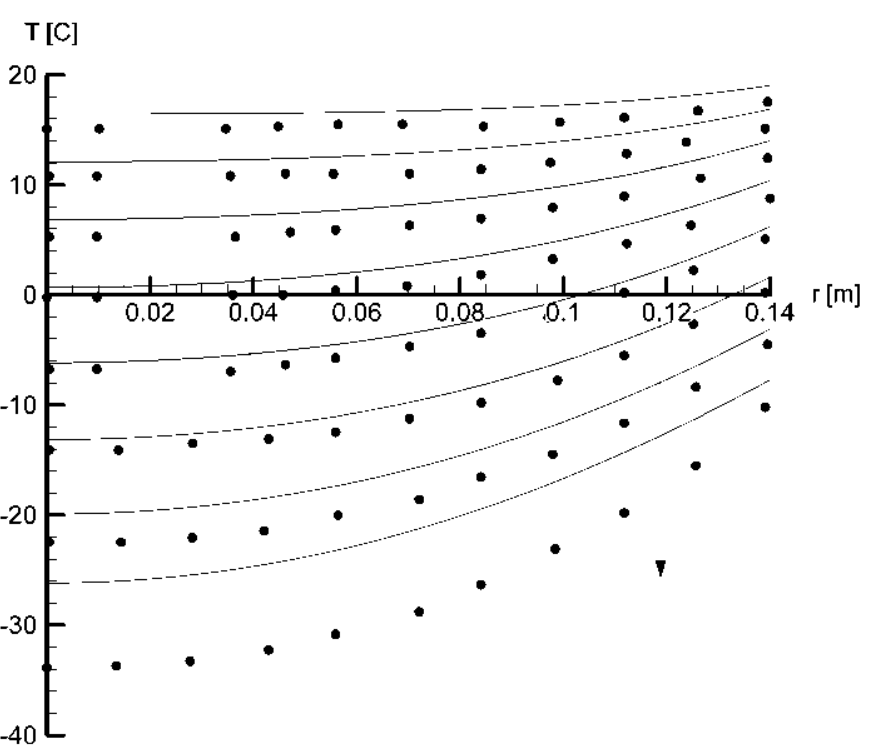

Fig. 3. Comparison of radial temperature profiles (GITT) at different times along the discharge (the arrow indicates increasing time steps), with the results of Mota et al. [5] (dotted). ( $t=0.25,0.5,0.75,1.0,1.25,1.5,1.75,2.0$ hours.)

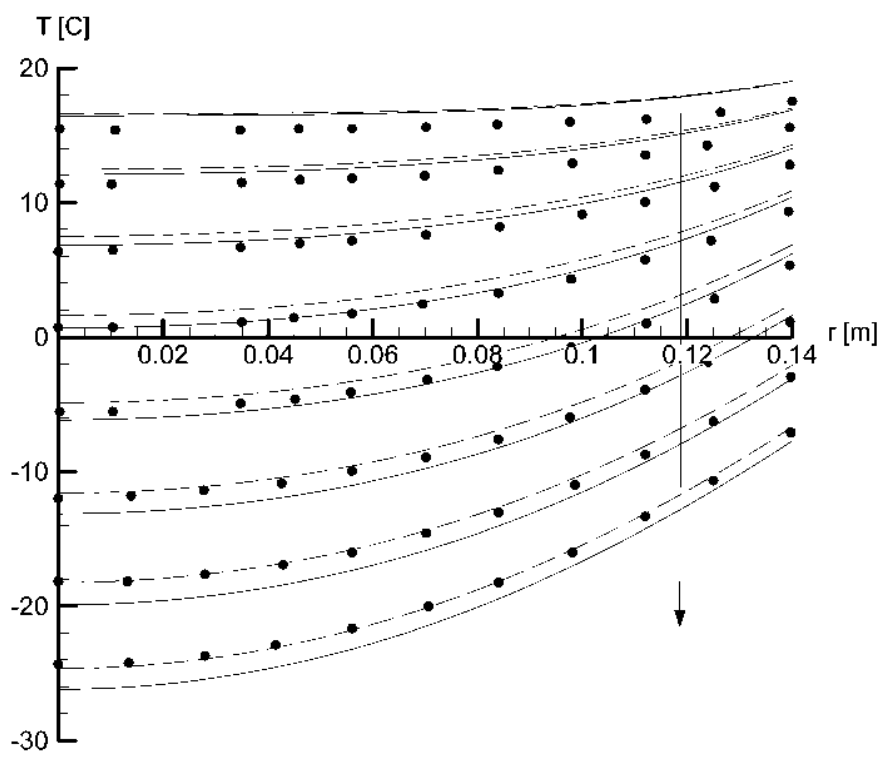

Fig. 4. Comparison of radial temperature profiles (GITT) at different times during discharge (the arrow indicates increasing time steps), without (dashed) and with (solid) prescribed pressure history, and the results of Couto et al. [9] (dotted). $(t=0.25,0.5,0.75,1.0,1.25,1.5,1.75,2.0$ hours.)

The present research effort has already resulted in the consolidation of a two-dimensional model for the ANG discharge process that includes the pressure evolution determination for an imposed mass flow rate and the adoption of a thermal control device at the center of the tank, such as a heat pipe, which was numerically solved in [19-21]. It is therefore of interest to covalidate these two implementations so as to provide a more definitive confidence on such simulation efforts. Fig. 4 presents the GITT results for the radial temperature profiles, for both the case of an imposed pressure gradient (solid), such as in Fig. 2, and for a constant mass flow rate (dashed), as chosen in Table 1 , when the pressure history is automatically determined by the algorithm. In addition, the results from [20] for a fixed mass flow rate are presented as dots, demonstrating the excellent agreement with the equivalent results by the Generalized Integral Transform Technique. It can also be noticed that the estimated pressure history from the plotted results
Table 1

Parameters used for code validation and solution methodology demonstration

\begin{tabular}{lll}
\hline Parameter & Mota et al. [5] & Chang and Talu [4] \\
\hline $\begin{array}{ll}\text { Activated carbon bed properties: } \\
\text { Type }\end{array}$ & G216 carbon pellets & N/A \\
Density $-\rho_{s}$ & $410 \mathrm{~kg} / \mathrm{m}^{3}$ & $975 \mathrm{~kg} / \mathrm{m}^{3}$ \\
Specific heat $-c_{p, s}$ & $650 \mathrm{~J} / \mathrm{kg} \mathrm{K}$ & $1052 \mathrm{~J} / \mathrm{kg} \mathrm{K}$ \\
Effective conductivity $-k_{\text {eff }}$ & $1.2 \mathrm{~W} / \mathrm{m} \mathrm{K}$ & $0.212 \mathrm{~W} / \mathrm{m} \mathrm{K}$ \\
Effective porosity $-\varphi_{\text {eff }}$ & 0.74 & 0.5 \\
Cylinder geometry: & & \\
Material & Stainless steel & Carbon steel \\
Density - $\rho_{w}$ & $7900 \mathrm{~kg} / \mathrm{m}^{3}$ & $7800 \mathrm{~kg} / \mathrm{m}^{3}$ \\
Specific heat $-c_{p, w}$ & $496.2 \mathrm{~J} / \mathrm{kg} \mathrm{K}$ & $502.4 \mathrm{~J} / \mathrm{kg} \mathrm{K}$ \\
Internal radius $-R_{\text {int }}$ & $0.14 \mathrm{~m}$ & $0.1 \mathrm{~m}$ \\
Length - $L$ & $0.85 \mathrm{~m}$ & $0.74 \mathrm{~m}$ \\
Thickness $-\delta_{w}$ & $0.01 \mathrm{~m}$ & $0.0055 \mathrm{~m}$ \\
Operational conditions: & & \\
Initial pressure $-P_{0}$ & $3.5 \mathrm{MPa}$ & $2.1 \mathrm{MPa}$ \\
Depletion pressure $-P_{d}$ & $0.1013 \mathrm{MPa}$ & $0.166 \mathrm{MPa}$ \\
Initial temperature $-T_{0}$ & $293 \mathrm{~K}$ & $291 \mathrm{~K}$ \\
Ambient temperature $-T_{\infty}$ & $293 \mathrm{~K}$ & $291 \mathrm{~K}$ \\
Heat transfer coefficient $-h_{\infty}$ & $4.0 \mathrm{~W} / \mathrm{m}^{2} \mathrm{C}$ & $\mathrm{N} / \mathrm{A} \mathrm{W} / \mathrm{m}^{2} \mathrm{C}$ \\
Flow rate $-\dot{m}$ & $0.0185 \mathrm{~kg} / \mathrm{min}{ }^{1}$ & $6.7 \mathrm{~L} / \mathrm{min}$ \\
Adsorption characteristics: & & \\
Heat of adsorption $-\Delta H$ & $-1.1 \times 10^{6} \mathrm{~J} / \mathrm{kg}$ & $\Delta H=f\left(R_{g}, \dot{m}\right)$ \\
\hline
\end{tabular}

1 Value inferred from performance coefficient data provided by Mota et al. [5].

$\mathrm{T}[\mathrm{C}]$

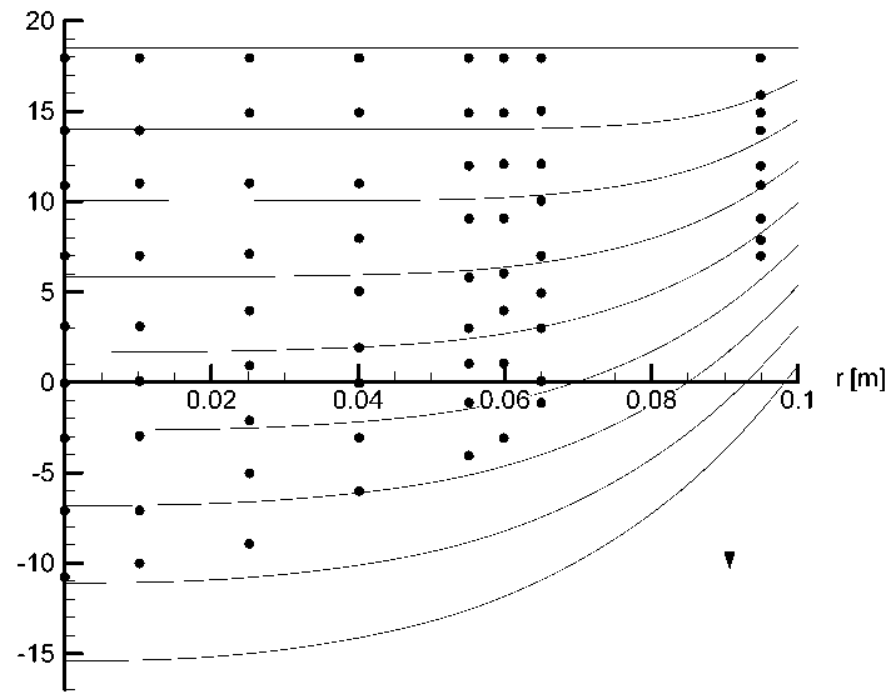

Fig. 5. Comparison of radial temperature profiles (GITT) at different times along the discharge (the arrow indicates increasing time steps), with the experimental data of Chang and Talu [4] (dotted). ( $t=0.25,0.5,0.75,1.0,1.25,1.5,1.75,2.0$ hours.)

of [5] still leads to reasonably accurate results for the temperature profiles, as illustrated by the dashed and solid lines, and that this aspect could not explain the increasing discrepancies between the present results and those of [5] for larger discharge times.

We now compare our results with the experimental data of [4], where a custom-built ANG test system was employed, and technical grade methane (99\%) was used in the experiments instead of natural gas. The values of the experimental parameters are listed in Table 1. As the authors did not provide the value of the convection heat transfer coefficient, we have used the value obtained from a convective heat transfer correlation for horizontal cylinders, which is about $4 \mathrm{~W} / \mathrm{m}^{2} \mathrm{~K}$. Fig. 5 shows a comparison of our GITT results against the experimental data of [4]. The data was collected every 20 minutes for a 3 hours discharge process. Larger values of the time variable show larger discrepancies between experiment and model, which predicts lower temperatures than experimentally measured, which might be due to the uncer- 


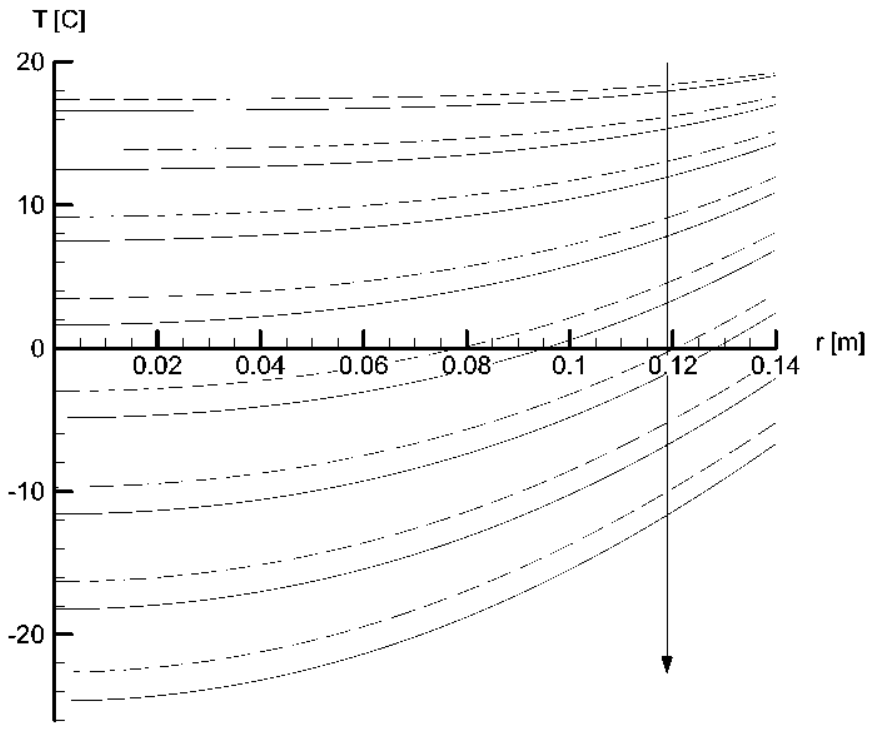

Fig. 6. Comparison of radial temperature profiles at different times during discharge (the arrow indicates increasing time steps), without (dashed) and with (solid) the inclusion of the pressure term on the energy equation. ( $t=$ $0.25,0.5,0.75,1.0,1.25,1.5,1.75,2.0$ hours.)

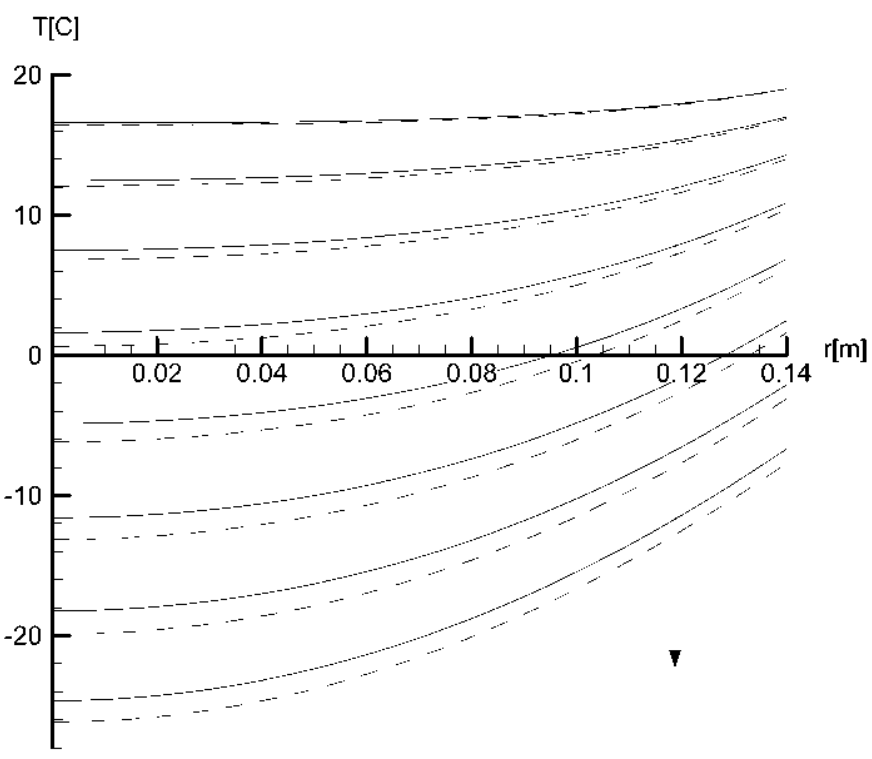

Fig. 7. Comparison of radial temperature profiles at different times during discharge (the arrow indicates increasing time steps), without (dashed) and with (solid) the inclusion of the energy accumulation term in the compressed and adsorbed gas phases. ( $t=0.25,0.5,0.75,1.0,1.25,1.5,1.75,2.0$ hours.)

tainty in the value adopted for the convection heat transfer coefficient. From this point on, all the parameter values used were those taken from Ref. [5], unless otherwise specified, and the influence of some effects that were previously considered negligible are here discussed. The first one is the pressure term in the energy equation, which accounts for the decompression work done by the gas, and as shown in Fig. 6 for the radial temperature profiles obtained with and without considering the pressure term, the decision of neglecting such term can lead to an overestimation of the temperature profiles. Also, as expected and illustrated in Fig. 7, again for the radial temperature distributions, it can be observed that neglecting the transient terms that account for the energy stored in the compressed and adsorbed phases induces the opposite effect of underestimating the temperature values. It can also be noticed that the importance of this term grows as the discharge time increases.

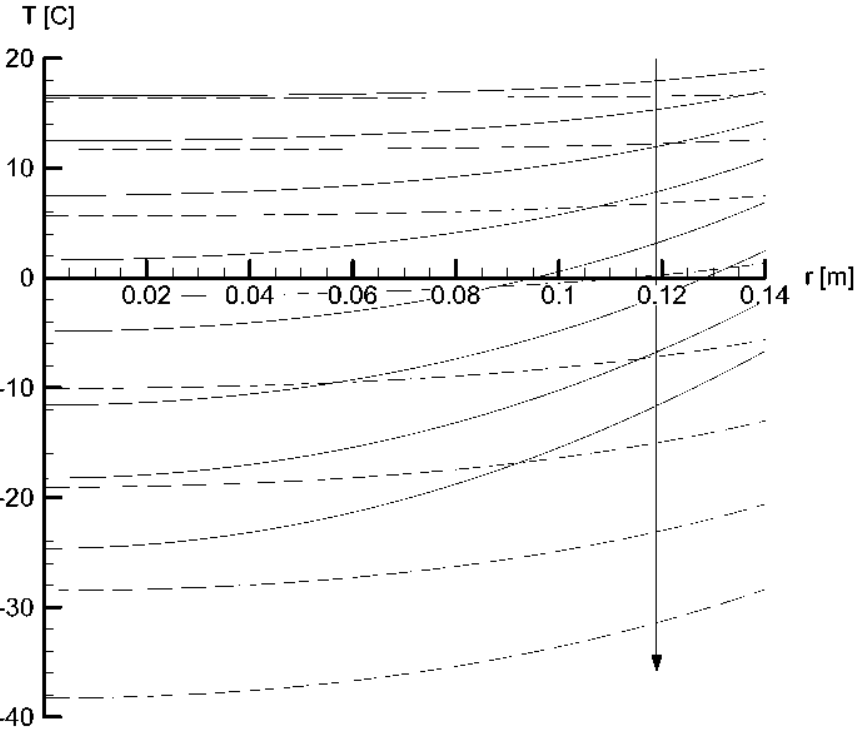

Fig. 8. Comparison of radial temperature profiles at different times during discharge (the arrow indicates increasing time steps), without (dashed) and with (solid) wall thermal capacitance effects. ( $t=0.25,0.5,0.75,1.0,1.25,1.5,1.75,2.0$ hours.)

We shall now analyze some heat exchange enhancement effects during discharge, towards the reduction of the adverse effect on storage capacity due to the heat of adsorption. We first conduct a critical comparison of the GITT solutions without and with wall conjugation effects, by reducing the wall thickness to a negligible value. In Fig. 8 we thus show radial temperature distributions for different degrees of wall conjugation, where the dashed lines are for the results without wall participation, and the solid lines are for the case where the wall thermal capacitance is accounted for. Clearly, the wall capacitance has a marked effect in slowing down the cooling of the porous bed along the slow discharge process, especially in regions closer to the wall. Even the center wall temperature increases in more than $10^{\circ} \mathrm{C}$ after 2 hours of discharge when considering the wall effect, suggesting the adoption of slender cylindrical tanks within the adequate mechanical constraints.

Another way of enhancing heat transfer, increasing the exchange area per unit volume of the cylinder, is to increase its aspect ratio $L / R_{\text {int. }}$ Fig. 9 shows the radial temperature profiles obtained with $L=1.7 \mathrm{~m}$ (dashed lines) and $L=0.85 \mathrm{~m}$ (solid lines), but for a fixed reservoir volume. For comparison purposes, the temperature profiles are shown as a function of the dimensionless radius. It can be observed that the increase of the aspect ratio due to a longer cylinder, causes a quite noticeable smoothing effect on the temperature distributions across the reservoir, with less marked temperature drops along the discharge period.

Enhancing the heat transfer rates to the surroundings can also reduce the temperature drop during discharge. As suggested by Mota et al. [5], this could be accomplished, for example, by forcing the exhaust gases that leave an engine at higher temperatures, to flow through a jacket around the reservoir. In order to analyse the influence of the wall heat transfer coefficient, we increase its value by two orders of magnitude. The variation of $h_{\infty}$ markedly influences the radial temperature profiles, as shown in Fig. 10, where the dashed lines represent the profiles obtained with $h_{\infty}=400$ $\mathrm{W} / \mathrm{m}^{2} \mathrm{~K}$ (forced convection), while the solid lines were obtained with $h_{\infty}=4 \mathrm{~W} / \mathrm{m}^{2} \mathrm{~K}$ (natural convection). As we increase $h_{\infty}$, the convection heat transfer is enhanced at the cylinder surface, inducing an augmentation of $21^{\circ} \mathrm{C}$ at the centre and of $27^{\circ} \mathrm{C}$ at the cylinder wall. Actually, for $h_{\infty}=400 \mathrm{~W} / \mathrm{m}^{2} \mathrm{~K}$, the wall temperature remains approximately with the same initial value throughout the discharge process. 
$\mathrm{T}[\mathrm{C}]$

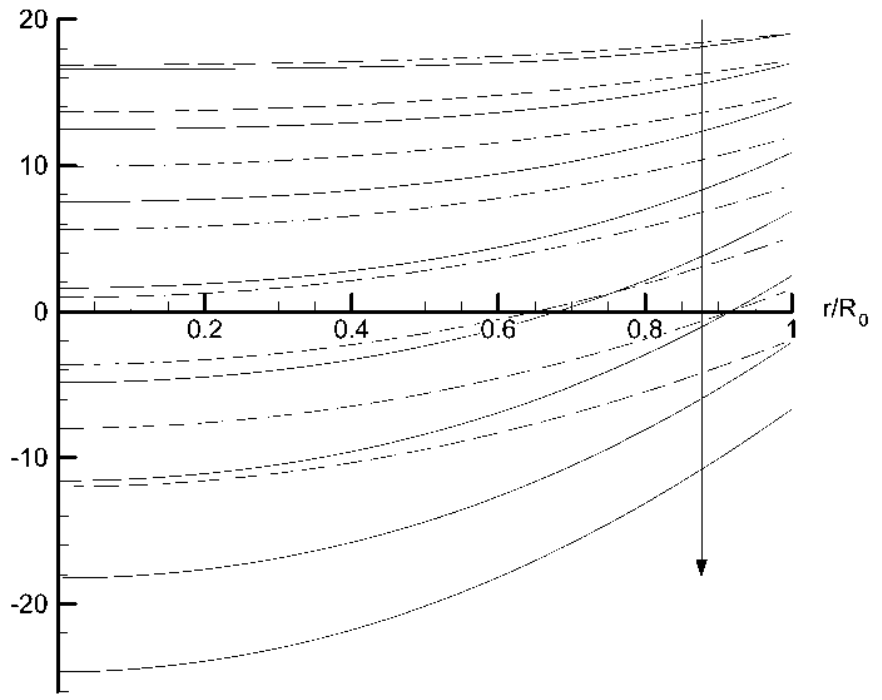

Fig. 9. Comparison of radial temperature profiles at different times during discharge (the arrow indicates increasing time steps), with $L=1.7 \mathrm{~m}$ (dashed) and $L=0.85 \mathrm{~m}$ (solid), for the same cylinder volume. $(t=0.25,0.5,0.75,1.0,1.25,1.5,1.75,2.0$ hours.)

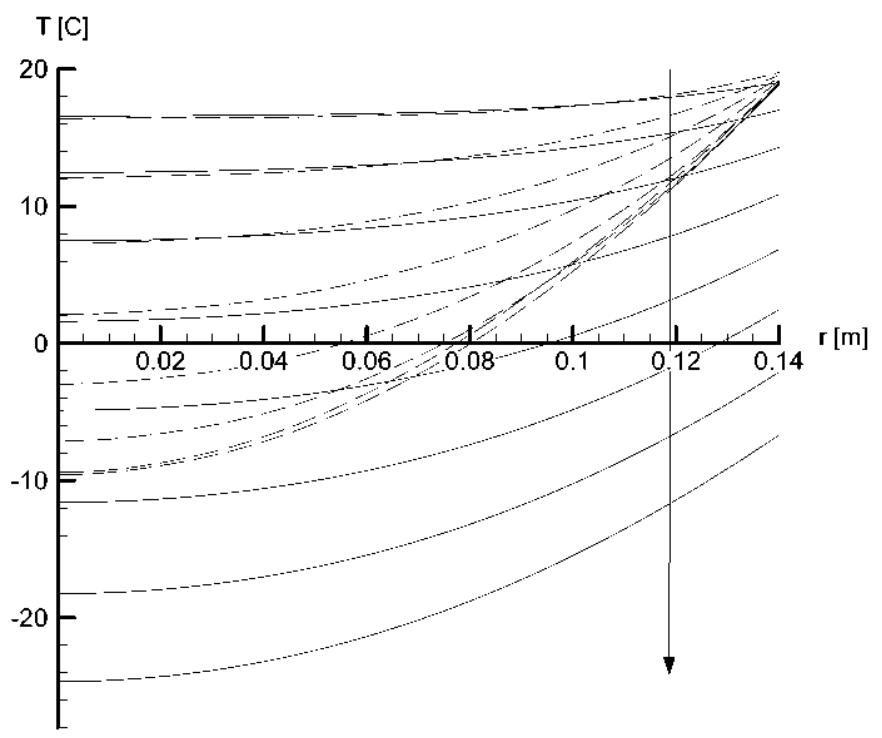

Fig. 10. Comparison of radial temperature profiles at different times during discharge (the arrow indicates increasing time steps), with $h_{\infty}=400 \mathrm{~W} / \mathrm{m}^{2} \mathrm{~K}$ (dashed) and $h_{\infty}=4 \mathrm{~W} / \mathrm{m}^{2} \mathrm{~K}$ (solid). ( $t=0.25,0.5,0.75,1.0,1.25,1.5,1.75,2.0$ hours.)

\section{Conclusions}

This work presented a one-dimensional transient nonlinear model for the slow discharge process of adsorbed methane cylinders, handled by a hybrid numerical-analytical solution based on the Generalized Integral Transform Technique (GITT). Natural gas was approximated as pure methane and modelled with an ideal gas thermodynamic behavior. The model accounts for the thermal capacity of the cylinder wall, convective heat transfer from the cylinder wall and the surrounding environment, and a prescribed mass flow rate leaving the cylinder.

The data obtained with the presented hybrid solution was compared to numerical data available in the literature [5] and the results of this comparison were encouraging, with the radial temperature profile presenting the same trends. The main difference between the present model and the one described by Mota et al.
[5] is that the later considered a known pressure history, while the former may also obtain the pressure history from the mass balance equation. This seems to be a more realistic condition as the consumption of natural gas from a cylinder is dictated by the power requirements of a thermal engine or cycle. The comparison with the finite differences solution of the two-dimensional transient model previously developed [18-21] revealed an excellent agreement. The model was then compared to the experimental results of [4], with the radial temperature profiles presenting the same trends. Discrepancies between the model and the experimental data are probably due to the lack of information about the value of the external heat transfer coefficient.

The influence of the terms that account for the decompression work done by the gas and the energy stored in the compressed and adsorbed phases was then investigated. Finally, the proposed solution was employed in the critical inspection of the wall thermal capacitance effect, of the influence of the aspect ratio of the cylinder as well as of the wall heat transfer coefficient with the external environment.

\section{Acknowledgements}

The authors wish to acknowledge the financial support provided by CNPq/CTPETRO, Brasil, through project no. 504816/04-7.

\section{References}

[1] T.A. Brady, M. Rostam-Abadi, M.J. Rood, Applications for activated carbons from waste tires: natural gas storage and air pollution control, Gas Separation and Purification 10 (10) (1996) 97-102.

[2] K. Inomata, K. Kanazawa, Y. Urabe, H. Hosono, T. Araki, Natural gas storage in activated carbon pellets without a binder, Carbon 40 (2002) 87-93.

[3] S. Biloé, V. Goetz, S. Mauran, Dynamic discharge and performance of a new adsorbent for natural gas storage, AIChE J. 47 (12) (2001) 2819-2830.

[4] K.J. Chang, O. Talu, Behaviour and performance of adsorptive natural gas storage cylinders during discharge, Appl. Thermal Engrg. 16 (5) (1996) 359-374.

[5] J.P.B. Mota, A.E. Rodrigues, E. Saatdjian, D. Tondeur, Dynamics of natural gas adsorption storage systems employing activated carbon, Carbon 35 (9) (1997) 1259-1270.

[6] L.L. Vasiliev, L.E. Kaninchik, D.A. Mishkinis, M.I. Rabetsky, Adsorbed natural gas storage and transportation vessels, Int. J. Thermal Sci. 39 (2000) 1047-1055.

[7] S. Biloé, V. Goetz, S. Mauran, Characterization of adsorbent composite blocks for methane storage, Carbon 39 (2001) 1653-1662.

[8] S. Biloé, V. Goetz, A. Guillot, Optimal design of an activated carbon for an adsorbed natural gas storage system, Carbon 40 (2002) 1295-1308.

[9] L.L. Vasiliev, L.E. Kanonchik, D.A. Mishkinis, M.I. Rabetsky, Adsorption systems of natural gas storage and transportation at low pressures and temperatures, J. Eng. Phys. Thermophys. 76 (5) (2003) 987-995.

[10] J.P.B. Mota, I.A.A.C. Esteves, M. Rostam-Abadi, Dynamic modelling of an adsorption storage tank using a hybrid approach combining computational fluid dynamics and process simulation, Computer \& Chem. Eng. 28 (2004) 24212431.

[11] O. Pupier, V. Goetz, R. Fiscal, Effect of cycling operations on an adsorbed natural gas storage, Chem. Eng. \& Processing 44 (2005) 71-79.

[12] M. Bastos-Neto, A.E.B. Torres, D.C.S. Azevedo, C.L. Cavalcante Jr., A theoretical and experimental study of charge and discharge cycles in a storage vessel for adsorbed natural gas, Adsorption 11 (2005) 147-157.

[13] R. Basumatary, P. Dutta, M. Prasad, K. Srinivasan, Thermal modeling of activated carbon based adsorptive natural gas storage system, Carbon 43 (2005) 541549.

[14] X.D. Yang, Q.R. Zheng, A.Z. Gu, X.S. Lu, Experimental studies of the performance of adsorbed natural gas storage system during discharge, Appl. Thermal Engrg. 25 (2005) 591-601.

[15] K.S. Walton, M.D. LeVan, Natural gas storage cycles: influence of nonisothermal effects and heavy alkanes, Adsorption 12 (2006) 227-235.

[16] F.N. Ridha, R.M. Yunus, M. Rashid, A.F. Ismail, Thermal transient behavior of an ANG storage during dynamic discharge phase at room temperature, Appl. Thermal Engrg. 27 (2007) 55-62.

[17] F.N. Ridha, R.M. Yunus, M. Rashid, A.F. Ismail, Dynamic delivery analysis of adsorptive natural gas storages at room temperature, Fuel Processing Tech. 88 (2007) 349-357.

[18] L.G. Lara, Theoretical analysis of the discharge process of adsorbed natura gas reservoirs, M. Sc. thesis, Mech. Eng. Program, COPPE, Fed. Univ. of Rio de Janeiro, Brazil, 2005 (in Portuguese). 
[19] L.G. Lara, P. Couto, R.M. Cotta, D.M.A. Sophia, Theoretical model for the discharge process of adsorbed natural gas reservoirs, in: 11th Brazilian Congress of Thermal Sciences and Engineering, ENCIT 2006 Curitiba, Brazil, Paper No. CIT06-0540, 2006

[20] P. Couto, L.G. Lara, D.M.A. Sophia, R.M. Cotta, Thermal control of adsorbed natural gas reservoirs under discharge dynamic condition, in: Proc. of the 13th Int Heat Transfer Conference Sidney, Australia, 2006.

[21] L.G. Lara, P. Couto, R.M. Cotta, D.M.A. Sophia, Gas discharge capacity intensification of adsorbed natural gas reservoirs, in: 11th Brazilian Congress of Thermal Sciences and Engineering, ENCIT 2006 Curitiba, Brazil, Paper No. CIT06-0633 2006.

[22] R.M. Cotta, Hybrid numerical-analytical approach to nonlinear diffusion problems, Numer. Heat Transfer, Part B 127 (1990) 217-226.

[23] R.M. Cotta, Integral Transforms in Computational Heat and Fluid Flow, CRC Press, Boca Raton, FL, 1993.
[24] R.M. Cotta, M.D. Mikhailov, Heat Conduction: Lumped Analysis, Integral Transforms, Symbolic Computation, Wiley-Interscience, New York, 1997.

[25] R.M. Cotta, M.D. Mikhailov, Hybrid methods and symbolic computations, in: W.J. Minkowycz, E.M. Sparrow, J.Y. Murthy (Eds.), Handbook of Numerical Heat Transfer, second ed., John Wiley, New York, 2006, pp. 493-522 (Chapter 16).

[26] R.M. Cotta, C.A.C. Santos, S. Kakaç, Unified hybrid theoretical analysis of nonlinear convective heat transfer, in: Proceedings of IMECE2007, ASME International Mechanical Engineering Congress \& Exposition, Seattle, Washington, USA, November 11-15, 2007, Paper No. IMECE2007-41412.

[27] R.M. Cotta, M.D. Mikhailov, Semi-analytical evaluation of integrals for the generalized integral transform technique, in: Proc. of the 4th Workshop on Integral Transforms and Benchmark Problems - IV WIT, CNEN, Rio de Janeiro, Brazil, 2005.

[28] S. Wolfram, The Mathematica Book, fourth ed., Wolfram Media, Cambridge, 1999. 